\title{
Tiotropium and exercise training in COPD patients: Effects on dyspnea and exercise tolerance
}

\author{
Nicolino Ambrosino' \\ Katia Foglio ${ }^{2}$ \\ Gianni Balzano² \\ Pier Luigi Paggiaro' \\ Patrizia Lessi ${ }^{3}$ \\ Steven Kesten ${ }^{3}$ \\ On behalf of the Tiotropium \\ Multicentric Italian Study Group \\ 'Cardio-Thoracic Department, \\ University Hospital, Pisa, Italy; \\ ${ }^{2} S$ Maugeri Foundation IRCCS, Italy; \\ ${ }^{3}$ Boehringer Ingelheim, GmbH, Milan, \\ Italy
}

\begin{abstract}
Background: Exercise training improves exercise tolerance in chronic obstructive pulmonary disease (COPD). Tiotropium $18 \mu \mathrm{g}$ once daily induces sustained bronchodilation throughout the day and reduces hyperinflation, one of the pathophysiological factors contributing to exertional dyspnea in COPD patients.
\end{abstract}

Aim: To determine whether tiotropium enhances the effects of exercise training in patients with COPD.

Design: Multicenter, 25 week randomized, double-blind, placebo-controlled, parallel-group study.

Setting: Twelve Italian Pulmonary Units practicing pulmonary rehabilitation.

Patients and intervention: Two hundred thirty four COPD patients (196 males; mean age: $67.4 \pm 7.6$; forced expiratory volume at 1 second $\left(\mathrm{FEV}_{1}\right): 41.4 \pm 13.0 \%$ predicted) were randomised to tiotropium $18 \mu \mathrm{g}$ or placebo inhalation capsules taken once daily. Both groups underwent a 8 week pulmonary rehabilitation program (PR) consisting of 3 exercise training session per week.

Measurements: Baseline, at the end of PR and after 12 weeks, patients completed pulmonary function testing, six minute walking test (6MWT), the Baseline and Transition Dyspnea Index (BDI and TDI), and the St. George's Respiratory Questionnaire (SGRQ).

Results: Relative to placebo, tiotropium had larger trough and post-study drug $\mathrm{FEV}_{1}$ responses on all test days. At the end of and 12 weeks following PR, patients on tiotropium showed no statistically significant differences in 6MWT compared to patients on placebo. Compared to the period immediately prior to PR, the mean improvement in 6MWT was only 29.7 meters (7.1\%) for the combined cohort. Mean TDI focal scores at the end of PR were 3.60 for tiotropium and 2.25 for placebo $(\mathrm{p}<0.01)$. At 12 weeks after PR, TDI focal scores were 2.71 for tiotropium and 2.11 for placebo $(\mathrm{p}=0.16)$. Reduction in all four SGRQ component scores, indicating an improvement in health-related quality of life, was observed for the tiotropium group over the duration of the study compared to placebo but the differences were not statistically significant. During the study period, there were fewer exacerbations and exacerbation days in the tiotropium group.

Conclusion: Although significant improvements were observed with perceived dyspnea, compared to placebo, the addition of tiotropium to pulmonary rehabilitation did not improve the 6MWT.

Keywords: bronchodilators, pulmonary rehabilitation, health-related quality of life, hyperinflation, dyspnea

\section{Introduction}

Chronic obstructive pulmonary disease (COPD) affects $6 \%$ of the general population and is a leading cause of morbidity and mortality and health-care costs worldwide (Mannino and Buist 2007). It is a heterogeneous disorder due to dysfunction of the small and large airways, as well as by destruction of the lung parenchyma and vasculature, in highly 
variable combinations (Celli et al 2004). Breathlessness and reduction in exercise capacity are the main complaints of COPD patients. These symptoms progress relentlessly reducing the patients' ability to perform activities of daily living (Pitta et al 2005). Different pathophysiological factors may contribute to exertional dyspnea in COPD patients: increased intrinsic mechanical loading on inspiratory muscles due to the intrinsic positive end-expiratory pressure, airflow limitation, inspiratory muscle weakness, increased ventilatory demand relative to capacity, gas exchange abnormalities, cardiovascular factors (Ambrosino and Serradori 2006). In COPD, exercise dyspnea correlates with lung hyperinflation, as assessed by inspiratory capacity, which, in turn, results in neuromechanical dissociation. Peripheral muscle dysfunction is also an important determinant of exercise capacity in COPD (Saey and Maltais 2005).

Moderate to very severe COPD patients may obtain improvement in dyspnea, exercise capacity, and healthrelated quality of life (HRQL) as a result of exercise training, a component of pulmonary rehabilitation (PR) (Nici et al 2006). The improvements in exercise tolerance induced by training have been found to be based on physiological changes, such as cardio-respiratory and peripheral muscle adaptations (Casaburi et al 1991; Maltais et al 1996) and are independent of the pattern of exercise-induced dynamic hyperinflation (Georgiadou et al 2007).

Tiotropium, an inhaled anticholinergic drug, provides sustained 24-hour improvement in airflow limitation and reduces hyperinflation with once-daily dosing (O’Donnell et al 2004). Data have also shown that tiotropium improves exercise tolerance, and HRQL (Aaron et al 2007). In addition, tiotropium in combination with PR improves exercise endurance time on treadmill, dyspnea and health status compared to PR alone; an effect sustained for 3 months following PR completion (Casaburi et al 2005).

The primary objective of the present study was to determine whether tiotropium, compared to placebo, enhances the improvement in exercise tolerance as measured by the six minute walk test (6MWT) in patients with COPD who participate in PR. The secondary objectives included an assessment of the impact of tiotropium on dyspnea and HRQL following PR. In addition, the study sought to evaluate whether the benefits after the conclusion of PR are better maintained with tiotropium.

\section{Materials and methods}

The study was approved by all local Independent Ethics Committees and was performed according to the Helsinki
Declaration; written informed consent was obtained from each patients prior to participation.

\section{Study design}

This was a 25 -week, multicenter, randomized, double-blind, placebo-controlled, parallel group study to evaluate the efficacy of tiotropium in patients with COPD participating in $\mathrm{PR}$, conducted in 12 sites in Italy. Patients were randomized 1:1 to either tiotropium $18 \mu \mathrm{g}$ qd or placebo for 25 weeks. Tiotropium or matching placebo were supplied as a dry-powder capsule delivered by the HandiHaler ${ }^{\circledR}$ device $^{2}$ (Boehringer Ingelheim; Ingelheim, Germany) and inhaled in the morning. The concomitant regular use of oral $(<10 \mathrm{mg} / \mathrm{d}$ of prednisone or equivalent) and inhaled steroids, short acting theophylline preparations, and mucolytic agents not containing bronchodilators was allowed during the study if the medication had been started for at least 6 weeks prior to the screening visit. Open-label salbutamol metered-dose inhaler was supplied during the study for as-needed acute symptom relief. Other $\beta_{2}$-agonists (long- and short-acting) and inhaled anticholinergic medications (other than study drugs) were not permitted.

\section{Patients}

Patients were required to have a clinical diagnosis of COPD (ATS 1995a), forced expiratory volume in one second $\left(\mathrm{FEV}_{1}\right) \leq 60 \%$ of predicted, and $\mathrm{FEV}_{1} \leq 70 \%$ of forced vital capacity (FVC). They were required to be at least 40 years of age and to have a smoking history ( $>10$ pack-years). Patients with a history of asthma, allergic rhinitis, eosinophils $\geq 8 \%$ were excluded from the study as well as patients with recent respiratory tract infections. Also, patients with significant diseases other than COPD or a recent history of myocardial infarction, hospitalisation for heart failure and cardiac arrhythmia requiring drug therapy, were not eligible for the trial. Other exclusion criteria included orthopedic, muscular, or neurologic disease that would interfere with regular participation in aerobic exercise or with exercise testing as well as a body mass index $(\mathrm{BMI})>30$ or $<18 \mathrm{~kg} \mathrm{~m}^{2}$. Patients who were in a PR program at the time of screening were also excluded.

\section{Study protocol}

The study protocol is described in Figure 1. During an initial screening visit (Visit 1), patients performed pulmonary function tests (PFTs) and performed the 6-minute walking test (6MWT). One week following the initial screening, patients were randomised to tiotropium or placebo for the subsequent 25 weeks. Following randomization, patients 


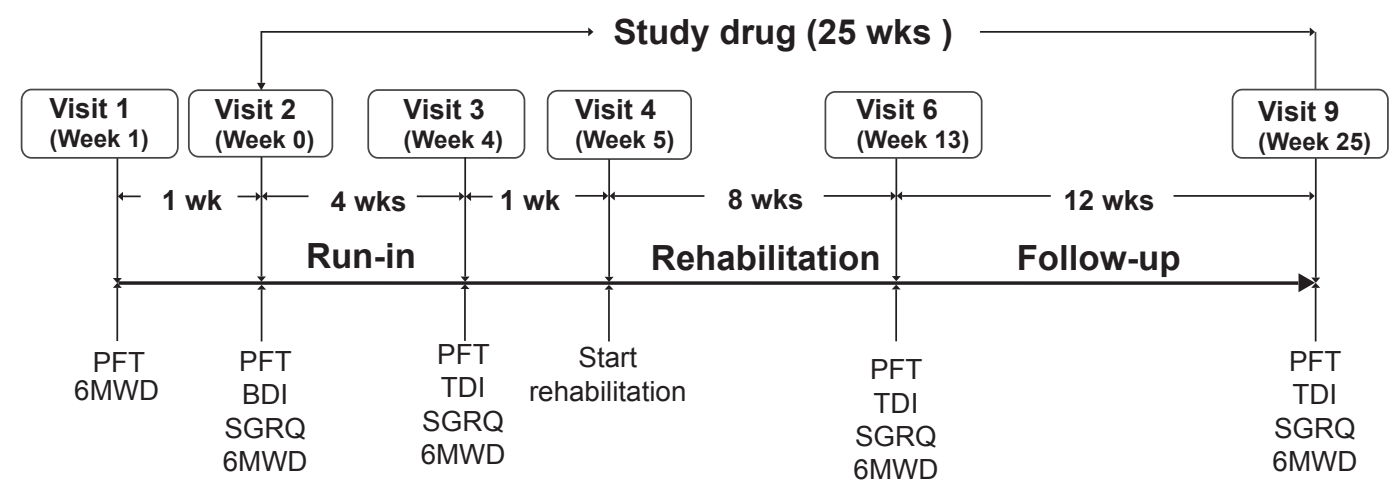

Figure I Outline of study protocol.

Abbreviations: BDI, baseline dyspnea index; PFT, pulmonary function test; SGRQ, St. George's Respiratory Questionnaire; 6MWT, 6 minute walk test.

entered a 4-week period during which they received study drug but not PR. PFTs and the 6MWT were repeated at the beginning (Visit 2) and end of this 4 week period (Visit 3). Patients then entered an 8-week PR program (Visit 4). After the last PR session (Visit 6), patients continued on study medication for a 12 week follow-up period. 6MWT were performed at the conclusion of the 8 weeks of PR (Visit 6) and after the 12 weeks of follow-up (Visit 9).

\section{Measurements}

\section{Lung function}

Spirometry was conducted according to the American Thoracic Society (ATS) criteria (ATS 1995b). The predicted values were calculated according to Quanjer (1983). The tests were performed at the screening visit; at randomisation (Visit 2); after 4 weeks of treatment (Visit 3); after PR (Visit 6) and after the 12-week follow-up period (Visit 9). Tests were performed prior to and 1 hour after dosing.

\section{Exercise}

Exercise tolerance was evaluated at the screening visit; at randomisation (Visit 2), which served as the baseline measurement; after 4 weeks of treatment (Visit 3); after PR (Visit 6) and after the 12-week follow-up period (Visit 9) by the 6MWT according to standard guidelines (ATS 2002). The best result of three tests was recorded. Except for Visits 1 and 2, all 6MWTs commenced 90 minutes after inhalation of study drug and approximately 15 to 30 minutes after spirometry. Arterial oxygen saturation was monitored by pulse oximetry throughout testing.

\section{Dyspnea}

Italian versions of the Baseline Dyspnea Index (Visit 2) and the Transition Dyspnea Index (TDI) (Mahler et al 1984) were used to assess dyspnea (Visits 3, 6 and 9). During the 6MWT, patients were asked to rate the intensity of dyspnea using the modified Borg scale every two minutes (Borg 1992).

\section{Health-related quality of life}

HRQL was evaluated by a validated Italian translation of the St. George's Respiratory Questionnaire (SGRQ) at Visits 2, 3, 6, and 9 (Carone et al 1999).

\section{Exacerbations and hospitalizations}

For the purpose of this study, exacerbations were defined as episodes (new onset or worsening of at least 2 respiratory symptoms) witha duration of 3 days or more requiring systemic steroids or antibiotics.

\section{Pulmonary rehabilitation program}

The lower limb exercise training protocols were performed on a treadmill 3 times weekly for 8 weeks. Initial treadmill speed was set to attain a targeted heart rate at $80 \%$ of the maximum heart rate obtained during the 6MWT at Visit 3. Dyspnea by the modified Borg scale, heart rate, pulse oximetry and treadmill speed was recorded every 10 minutes. Training was maintained for as long as tolerated for at least 30 minutes. For patients unable to complete 30 minutes of continuous lower limb exercise, exercise training sessions could be divided into as many as 4 parts with 5 to 10 minute rests in between.

Other exercise modalities were encouraged. All patients were encouraged to exercise at home on days when they were not attending the centre. Pulmonary rehabilitation included also upper limb activities as well as patient education. The form of these activities were determined by each centre and was not specified within the protocol.

\section{Statistical analysis}

For the primary analysis, a full analysis set (FAS) that included all patients randomized and treated with a baseline 
6MWT value and at least one 6MWT value on treatment available, was considered. In order to be able to include the same patients at each time point in the 6MWT summaries, missing post baseline values caused by a patient's intake of rescue medication or a patient's early discontinuation due to worsening of COPD disease were replaced by the minimum value at the previous visits. For missing values due to other reason were replaced by the median of all nonmissing distance on that visit. The two treatments groups were compared using an analysis of covariance (ANCOVA) model that included terms for center and treatment as main effects and baseline (data at Visit 2) as a linear covariate. Interactions: centers-by-treatment and treatment-by-baseline were also tested adding appropriate terms to the main ANCOVA model.

Treatment differences are presented as least square means \pm standard error (SE) and corresponding $p$-values taken from ANCOVA. Because of the a pre-specified null hypothesis testing procedure (at a 2 -sided $\alpha$ level of 0.05 ) on a single primary variable, no adjustment for multiplicity was required. A sensitivity analysis was performed on the primary variable considering the per protocol set (PPS) that included patients in FAS and without important protocol violations.
The spirometry measurements $\left(\mathrm{FEV}_{1}\right.$ and FVC), TDI scores and SGRQ scores were analysed on the FAS using the same main model specified for the primary endpoint with imputation for missing data.

Continuous baseline characteristics are shown as mean \pm standard deviation (SD) and were compared between the two treatment groups by using the Mann-Whitney-Wilcoxon test or when appropriate the t-test. Categorical baseline characteristics are expressed as counts and percentages and were compared between groups by using the chi-squared test.

\section{Sample size}

A sample of 182 patients (91 per treatment group) was calculated as adequate to detect a 54 meter (Redelmeier et al 1997). difference between tiotropium and placebo at $5 \%$ significance level, with $80 \%$ power using a two-tailed t-test. Assuming a $20 \%$ attrition rate, the total planned sample size was 220 . All the statistical analyses were performed using the SAS package (version. 8.2, SAS Inc., Cary, NC, USA).

\section{Results}

Patients recruited, eligible withdrawals and completions are shown in Figure 2. Overall, 177 patients (87 tiotropium,

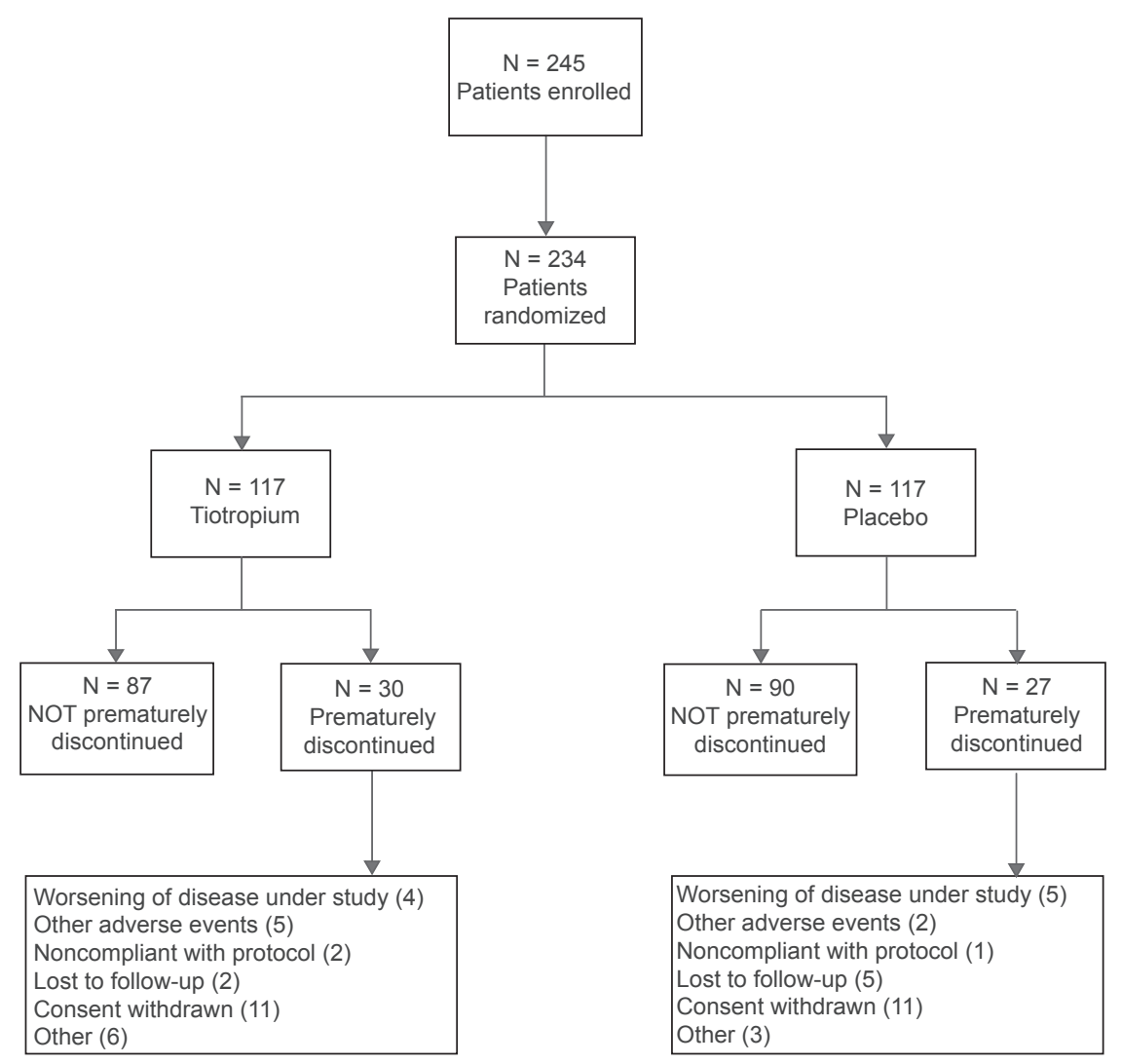

Figure 2 Patients flow diagram. 
90 placebo) completed the trial according to the protocol. Of the 57 patients prematurely discontinued, 30 (25.6\%) had been randomized to tiotropium and $27(23.1 \%)$ to placebo. The reasons for discontinuation are also shown in Figure 2 was consent withdrawn ( $9.4 \%$ of treated patients). The FAS included 103 patients in the tiotropium group and 106 patients in the placebo group. There were 154 (75 on tiotropium, 79 on placebo) patients in the PPS.

At study entry, the patients' baseline characteristics were balanced between the two groups (Table 1). The percentage of patients taking any pulmonary medication at baseline period was nonsignificantly higher in the placebo than in the tiotropium group $(60.7 \%$ vs $53.0 \%, \mathrm{p}=0.24)$. At baseline $23.9 \%$ of patients in the placebo and $14.5 \%$ in the tiotropium group were receiving inhaled anticholinergics. The percentage of patients taking any pulmonary medication during the treatment period (other than that supplied as part of the trial) was $54.7 \%$ for both groups. The use of inhaled steroids during the treatment period was $41.9 \%$ for the tiotropium vs $52.1 \%$ for the placebo group $(p=0.12)$. The proportion of patients performing either arm exercise or exercising at home was similar for both groups.

\section{Lung function}

Tiotropium significantly increased post-dose $\mathrm{FEV}_{1}$ at week 13 and 25 compared to placebo (adjusted mean differences (AMD) between groups $0.103 \pm 0.052 \mathrm{~L}, 0.122 \pm 0.052 \mathrm{~L}$ and $0.110 \pm 0.049 \mathrm{~L}$ on test week 4, 13 and 25, respectively; $\mathrm{p} \leq 0.05$ on all test days).

Patients receiving tiotropium had larger trough $\mathrm{FEV}_{1}$ responses than patients receiving the placebo on all the test days (AMD between groups: $0.100 \pm 0.05 \mathrm{~L}$ on test week 4; $\mathrm{p}=0.047)$. The trough AMD on the other two test days were not statistically significant.

Tiotropium significantly increased post-dose FVC on all test days compared to placebo (AMD between groups: $0.154 \pm$ $0.064 \mathrm{~L}, 0.176 \pm 0.067 \mathrm{~L}$ and $0.121 \pm 0.061 \mathrm{~L}$ on the test days 29, 92 and 176, respectively; $\mathrm{p}<0.05$ on all test days) Tiotropium increased trough FVC compared to placebo (AMD between groups: $0.124 \pm 0.061 \mathrm{~L}, 0.125 \pm 0.065 \mathrm{~L}$ and $0.102 \pm$ $0.063 \mathrm{~L}$ on test week 4, 13, and 25, respectively, the difference on test week 4 being statistically significant: $\mathrm{p}=0.044$ ).

\section{Exercise tolerance}

The adjusted means of 6MWT on each test day are presented in Figure 3. At Visit 3, after 29 days of treatment, patients

Table I Baseline characteristics of patients in study*

\begin{tabular}{|c|c|c|c|c|}
\hline \multirow[t]{2}{*}{ Total treated $[\mathrm{N}]$} & Placebo & Tiotropium & All & p-value \\
\hline & 117 & 117 & 234 & \\
\hline Male [N (\%)] & $99(84.6)$ & $97(82.9)$ & $196(83.7)$ & $0.72^{\circ}$ \\
\hline Age (yrs) & $66.9 \pm 7.3$ & $67.8 \pm 7.8$ & $67.4 \pm 7.6$ & $0.38^{\S}$ \\
\hline Duration of COPD (yrs) & $11.3 \pm 9.5$ & $10.9 \pm 9.8$ & $11.1 \pm 9.6$ & $0.66^{\#}$ \\
\hline BMI $\left(\mathrm{kg} / \mathrm{m}^{2}\right)$ & $26.7 \pm 4.0$ & $26.4 \pm 4.0$ & $26.6 \pm 4.0$ & $0.57^{\S}$ \\
\hline Smoking history - pack/years & $35.0 \pm 22.4$ & $38.3 \pm 25.2$ & $36.7 \pm 23.8$ & $0.33^{\#}$ \\
\hline $\mathrm{FEV}_{1}(\mathrm{~L})$ & $\mathrm{I} . \mathrm{I} \pm 0.4$ & $\mathrm{I} . \mathrm{I} \pm 0.4$ & $1.1 \pm 0.4$ & $0.62^{\S}$ \\
\hline FEV $(\%$ predicted $)$ & $40.3 \pm 12.6$ & $42.5 \pm 13.3$ & $41.4 \pm 13.0$ & $0.20^{\S}$ \\
\hline $\mathrm{FEV}_{1} / \mathrm{FVC}(\%)$ & $45.2 \pm 10.4$ & $47.3 \pm 11.8$ & $46.3 \pm 11.2$ & $0.15^{\S}$ \\
\hline $\mathrm{FVC}(\mathrm{L})$ & $2.5 \pm 0.8$ & $2.4 \pm 0.7$ & $2.5 \pm 0.8$ & $0.54^{\S}$ \\
\hline 6MWT (meters) & $402 \pm 119.5$ & $404.8 \pm 115.6$ & $403.4 \pm 117.3$ & $0.86^{\S}$ \\
\hline \multicolumn{5}{|l|}{ Baseline pulmonary medication ${ }^{\dagger}$ : } \\
\hline Total taking pulmonary medication [N (\%)] & $71(60.7)$ & $62(53.0)$ & $133(56.8)$ & $0.23^{\circ}$ \\
\hline Anticholinergics (Short-acting/inhaled) & $28(23.9)$ & $17(14.5)$ & $45(19.2)$ & $0.07^{\circ}$ \\
\hline Beta-adrenergics (Long-acting/inhaled) & $28(23.9)$ & $13(11.1)$ & $4 \mid(17.5)$ & $0.01^{\circ}$ \\
\hline Beta-adrenergics (Oral/short-acting/inhaled) & $39(33.3)$ & $32(27.4)$ & $71(30.3)$ & $0.32^{\circ}$ \\
\hline Steroids (Oral/inhaled/IV/IM) & $60(51.3)$ & $44(37.6)$ & $104(44.4)$ & $0.04^{\circ}$ \\
\hline Xanthines & $28(23.9)$ & $28(23.9)$ & $56(23.9)$ & $1.0^{\circ}$ \\
\hline
\end{tabular}

Notes: *Data are presented as mean \pm standard deviation unless otherwise indicated; ${ }^{\circ} \mathrm{p}$-value based on chi-squared test; ${ }^{\S} \mathrm{p}$-value based on t-test; ${ }^{\#} \mathrm{p}$-value based on Mann-Whitney-Wilcoxon test; ${ }^{\dagger}$ Only frequent (>10\%) pulmonary medication were reported.

Abbreviations: BMI, body mass index; COPD, chronic obstructive pulmonary disease; FEV , forced expiratory volume in one second; FVC, forced vital capacity. 


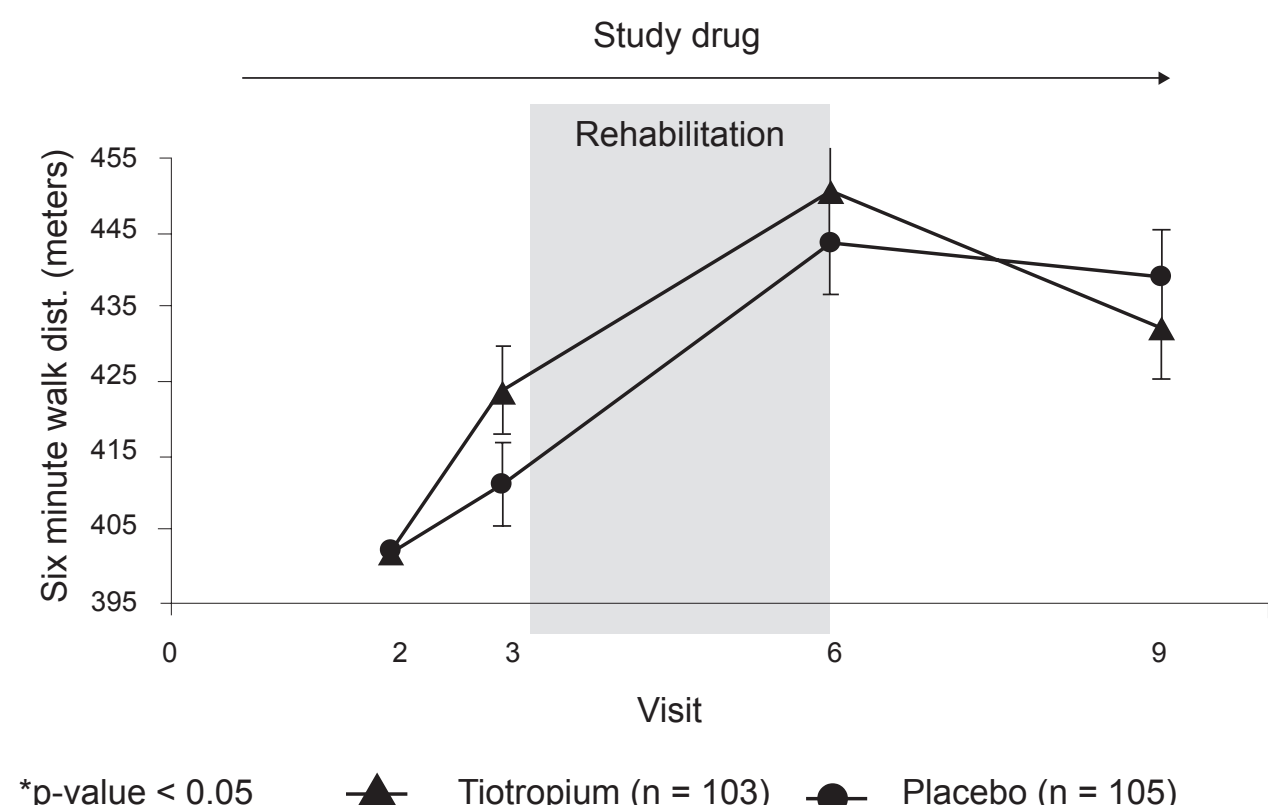

Figure 3 Adjusted means (SE) of 6 minute walk test distance (meters) on each visit for the tiotropium and placebo groups.

on tiotropium walked nonsignificantly longer than those on placebo $(423.8 \pm 5.9$ vs 411$) .1 \pm 5.7$ meters respectively: AMD between groups: $12.7 \pm 8.0$ meters, $95 \%$ confidence interval $[\mathrm{CI}]-3.1$ to $28.5, \mathrm{p}=0.12)$. After 13 weeks of treatment, including 8 weeks of PR (Visit 6, day 92), patients on tiotropium showed a nonsignificant longer walk distance compared to those on the placebo $(450.8 \pm 7.0 \mathrm{vs}$ $443.5 \pm 6.8$ meters, respectively, AMD: $7.3 \pm 9.5$ meters, $95 \% \mathrm{CI}-11.4$ to $26.1, \mathrm{p}=0.44)$. Compared to the period immediately prior to $\mathrm{PR}$, the mean improvement in 6MWT was only 29.7 meters (7.1\%) for the combined cohort. After 25 weeks of treatment, 12 weeks after the termination of the PR (Visit 9, day 176), patients in both groups showed a reduction in exercise capacity without any significant difference between groups (AMD $-6.8 \pm 9.3$ meters, 95\% $\mathrm{CI}-25.1$ to $11.5, \mathrm{p}=0.46)$.

The primary analysis was also performed on the PPS data set. Patients on tiotropium walked longer distance on all the test days. The AMD between the two groups on Visit 3 (day 29) was $21.0 \pm 8.6$ meters, 95\% CI 4.0 to 37.9 , $\mathrm{p}=0.02$. The AMD on the other two test days (92 and 176) were $13.2 \pm 11.3$ and $5.1 \pm 10.9,(p=0.24$ and $p=0.64$ respectively).

\section{Dyspnea}

The mean BDI score was 6.6 units for both treatment groups. As shown in Figure 4 from test day 29 to 92 (at the end of PR), both treatment groups showed increases in the mean TDI focal scores, with the tiotropium group showing a larger increase $(2.25 \pm 0.29$ vs $3.60 \pm 0.30, \mathrm{p}=0.001)$ and exceeded the minimal clinically important difference (MCID) of 1 unit. The differences on the test days 29 and 176 were not statistically significant. Of note, both the placebo and tiotropium treatment groups improved by more than 1 unit at day $29(1.21 \pm 0.22$ vs $1.45 \pm 0.23, \mathrm{p}=0.44)$.

\section{Health-related quality of life}

The SGRQ total score improved after treatment and then remained stable in both groups, and trended towards a lower score on each test day in tiotropium compared to placebo group, which did not reach statistical significance (Figure 5). The mean change from baseline was -2.1 units (37.0 vs 39.1) in the placebo group and -3.5 units (35.6 vs 39.1 ) in the tiotropium group at day 29. The changes from baseline to day 92 were -6.1 units (33.1 vs 39.1 ) in the placebo group and -8.1 units (30.9 vs 39.1) in the tiotropium group. The changes on day 176 were -6.0 units (33.1 vs 39.1 ) and -7.3 units (31.8 vs 39.1 ) in the placebo and tiotropium groups, respectively. Improvements in both groups exceeded the MCID of 4 units at the end of PR and the end of the follow-up period.

\section{Exacerbations and hospitalizations}

The proportion of patients suffering from at least one COPD exacerbation was $13.6 \%$ (14/103 patients) in the tiotropium and $19.8 \%(21 / 106$ patients) in the placebo group, with a relative risk reduction of $31.3 \%(\mathrm{p}=0.23)$. The total number of COPD exacerbations were 19 in the tiotropium and 26 in 


\section{Study drug}

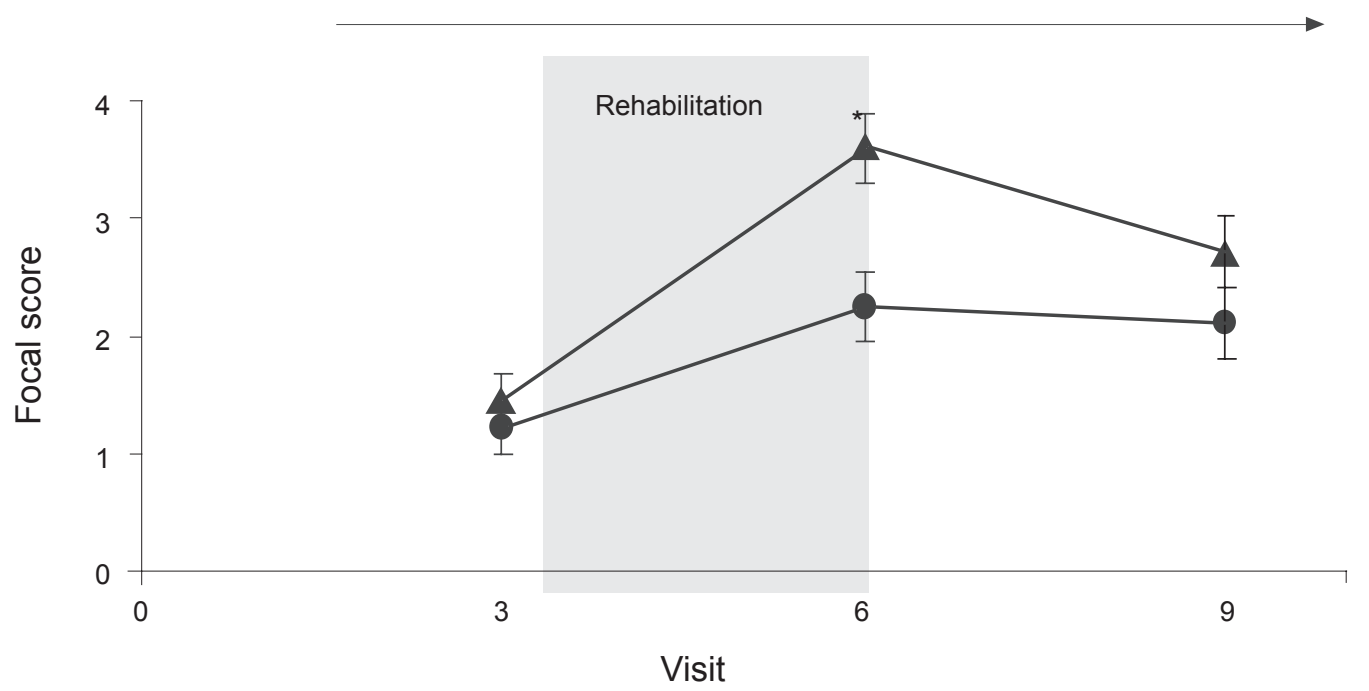

${ }^{*}$ p-value $<0.05 \quad$ Tiotropium $(n=103) \quad-\quad$ Placebo $(n=105)$

Figure 4 Adjusted means (SE) of the transition dyspnea index focal score on each visit for the tiotropium and placebo groups.

the placebo group. The total COPD exacerbation days were 318 in the tiotropium and 361 in the placebo group $(p=0.21)$. There were 2 COPD exacerbations leading to hospitalizations in tiotropium and 6 in the placebo group.

\section{Discussion}

In the present clinical trial, compared to placebo, tiotropium in combination with pulmonary rehabilitation significantly improved dyspnea (day 92) but did not show any additional benefit to PR in exercise tolerance, as measured by the 6MWT. Relatively minor changes were observed in the 6MWT in both groups with PR. There were clinically meaningful improvements in dyspnea (ie, TDI focal score $>1$ unit) in both groups prior to, which improved further after PR. Improvement in HRQL was larger in tiotropium than in placebo group although differences were not statistically significant. In both groups, large changes in the SGRQ total score were observed after PR which were relatively well maintained during the 12 week follow-up period.

Evidence-based support for PR in the management of COPD patients has accumulated in the last decade, and this comprehensive intervention has been unequivocally demonstrated to reduce dyspnea, increase exercise performance, and improve HRQL. Furthermore, an emerging literature is beginning to reveal its effectiveness in reducing health care costs (Nici et al 2006). The hypothesis of this study was that in terms of aerobic conditioning, there might be an advantage of tiotropium administered during a period of exercise training. Tiotropium induces sustained bronchodilation throughout the day and has superior spirometric outcomes in comparison to placebo or ipratropium bromide. In addition, several studies demonstrated sustained reductions in hyperinflation with tiotropium (O’Donnell et al 2004; Maltais et al 2005). These outcomes might be enough to allow patients to exercise longer or with a higher intensity thereby leading to improved exercise tolerance (Maltais et al 2005). Furthermore, the benefits gained may have a longer lasting effect due to the higher exercise tolerance attained. However, the relatively modest improvement in exercise tolerance following PR in both groups in the present study appears to be contrary to those of Casaburi et all (Casaburi et al 2005).

The explanation for the modest improvement in exercise capacity to either PR or to tiotropium in the present study likely relates to methodologic issues including the choice of functional measurement of endurance. A similar rationale also can explain the differing results between the present trial and the Casaburi study (Casaburi et al 2005). In the report by Casaburi, the effect of tiotropium on exercise tolerance in patients with COPD participating in 8 weeks of PR was measured by endurance time during a constant work rate treadmill exercise test, whereas in our study we used the 6MWT. Although strong relationships between 


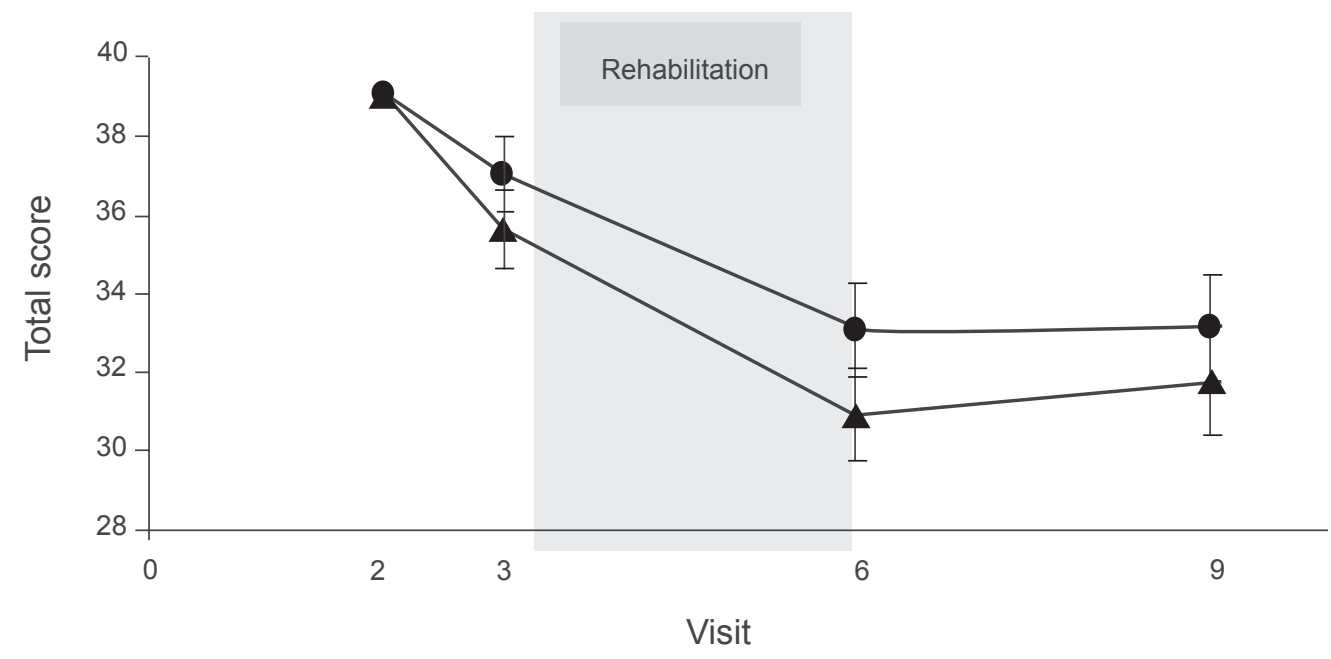

${ }^{*}$ p-value $<0.05 \quad$ Tiotropium $(n=103) \quad$ Placebo $(n=104)$

Figure 5 Adjusted means (SE) of the St. George's Respiratory Questionnaire total score on each visit for the tiotropium and placebo groups.

different methods of exercise assessment have been reported (Turner et al 2004), the two methods evaluate aspects somehow different of exercise, which may explain the differences in results between the two studies. The relative responsiveness of the 6MWT to detect changes in exercise performance following bronchodilation and PR appears to be lower than other forms of exercise testing. Pepin and colleagues (2007) explored the sensitivity of the 6MWT to acute bronchodilation compared with the endurance shuttle walk test. Despite demonstrating acute spirometric improvements, the 6MWT distance did not improve; however, improvements were evident in the shuttle walk test.

The results probably were likely influenced by patient selection. At baseline, the patients had a relatively well preserved 6MWT (mean $>400$ metres), which could have potentially led to ceiling effect. Another explanation includes variability in the conduct of the test due to the enrolment of multiple sites with variable experience. For example, despite training in the conduct of the study, several centres did not adhere performance of protocol mandated. As well, many centres tended to round the 6MWT results to the closest 10 meter value rather than determine the single digit result. There was also variability in the physical construction of the test (ie, variability in the length of the corridor).
Twelve weeks after the termination of PR, patients in both groups showed a reduction in exercise capacity without any significant difference between groups. Limited studies suggest that the benefits of PR decline toward baseline after 6 to 12 months, but remain improved compared with control subjects after 1 year (Foglio et al 1999).

The modest changes observed in the 6MWT appeared to be contrary to the clinically meaningful changes in the TDI focal score. The tiotropium group reported the lowest perception of dyspnea. It might therefore be assumed that tiotropium treated patients could engage in a longer duration and/or more intense physical activity compared to the placebo group. In a subgroup analysis of the study initially reported by Casaburi and colleagues (2005), tiotropium appeared to increased patient self-reported participation in physical activities (Kesten et al 2008). In the present study from test week 4 to 13 both treatment groups showed increases in the TDI scores, indicating an improvement in dyspnea, with the tiotropium group showing a significantly larger increase on test week 13 (after the PR period), but not on test week 4 and test week 25. The difference in the TDI focal score from placebo at the conclusion of PR (day 92) exceeded the MCID of 1 unit. It has been suggested that the categorisation of patients with COPD according to the level of dyspnea is more discriminating than staging of disease severity on the basis of airway obstruction with respect to 5-year survival (Nishimura et al 2002). 
The observed apparent dissociation of 6MWT and dyspnea was also observed for the SGRQ. In both groups, PR resulted in rather dramatic improvements in SGRQ total score that exceeded the MCID of 4 units. The SGRQ total score was lower on each test day in the tiotropium compared to the placebo group although the differences were not statistically significant. As opposed to the 6MWT, the SGRQ remained stable following PR (Figure 4). This observation has been reported also in other studies (Foglio et al 1999, 2007) and probably reflects the comprehensive nature of the PR and the fact that health status depends on more than just exercise capacity. Studies in COPD patients using both the Chronic Respiratory Questionnaire and SGRQ (Ketelaars et al 1996; Tsukino et al 1996) have shown that HRQL is only partially determined by lung function and exercise tolerance. Therefore, HRQL should be specifically evaluated in addition to physiological measures before and after PR. Furthermore it has been reported that generic and disease specific HRQL are independent risk factors for respiratory and all-causes mortality (Domingo-Salvany et al 2002).

The present study documented improvements in spirometry and a trend towards reduced exacerbations. While spirometry is not expected to improve with PR, it was expected that tiotropium would show similar improvements to other published reports (O’Donnell et al 2004; Maltais et al 2005). Patients receiving tiotropium had larger trough $\mathrm{FEV}_{1}$ and $\mathrm{FVC}$ responses than those receiving placebo on all the test days, although the mean difference between the tiotropium and the placebo group was significant only on test day 29. Tiotropium significantly increased patients post-dose $\mathrm{FEV}_{1}$ and FVC on all the test days compared to placebo. These improvements were higher than those observed in the study by Casaburi and colleagues (2005) in which more severe patients were enrolled. The lack of statistically significant differences in trough values at test days 92 and 176 between the two treatments groups was unexpected and could be explained by a larger variability observed in spirometry outcomes in the present study.

In summary, tiotropium in combination with PR significantly improved dyspnea at the end of the program but did not show any additional benefit in the 6MWT, compared to PR and placebo. We encountered methodological issues regarding the $6 \mathrm{MWT}$ in the present study. The relatively modest changes in the 6MWT with PR in the trial suggest that the use of this field test in multicenter clinical trials may have limitations not fully understood or reported. Alternative tests of functional capacity should be considered in multicenter COPD clinical trials.

\section{Acknowledgments}

The authors thank the following investigators and their research staff who participated in this trial. The authors also thank Dr Paolo Serra (Hyperphar Group; Milan) for assistance with statistical analysis. The study was funded by Boehringer Ingelheim Italy (Milan, Italy) and Pfizer Italy (Rome, Italy).

\section{Study investigators}

G. Balzano (S Maugeri Foundation, Telese); G. Barisione (San Martino Hospital, Genova); A. Cogo (University of Ferrara, Ferrara); M. Confalonieri (Santorio Hospital, Trieste); G. De Angelis (San Camillo Hospital, Roma); K. Foglio (S Maugeri Foudation, Lumezzane); F. Gigliotti (Don Gnocchi Foundation, Pozzolatico); A. Meriggi (S Maugeri Foundation, Casorate Primo); M. Neri (S Maugeri Foundation, Tradate); P.L. Paggiaro (Pisa University, Pisa); F. Pasqua (San Raffaele Hospital, Velletri); A. Spanevello (S Maugeri Foundation, Cassano delle Murge).

\section{Disclosure}

Katia Foglio and Gianno Balzano have no potential conflict of interest. Nicolino Ambrosino and Pierluigi Paggiaro accepted the following funds from an organization that may in any way gain or lose financially from the results of this study: fees for speaking, organizing education, and consulting, and funds for research. Patrizia Lessi, and Steven Kesten are employed by an organization that may in any way gain or lose financially from the results of this study.

BI Trial n 205 247. The study was funded by Boehringer Ingelheim Italy (Milan, Italy) and Pfizer Italy (Rome, Italy).

\section{References}

[ATS] American Thoracic Society. 1995. Standardization of Spirometry, 1994 Update. Am J Respir Crit Care Med, 152:1107-36.

[ATS] American Thoracic Society. 1995. Standards for the diagnosis and care of patients with chronic obstructive pulmonary disease (COPD) and asthma. Am Rev Respir Dis, 152:S77-S120.

[ATS] American Thoracic Society. 2002. ATS Statement: Guidelines for the Six-Minute Walk Test. Am J Respir Crit Care Med, 166:111-7.

Aaron SD, Vandemheen KL, Fergusson D, et al. 2007. Tiotropium in combination with placebo, salmeterol, or fluticasone-salmeterol for treatment of Chronic Obstructive Pulmonary Disease. A randomized trial. Ann Intern Med, 146:545-55.

Ambrosino N, Serradori M. 2006. Dyspnea, linguistic and biological descriptors. Chron Respir Dis, 3:117-22.

Borg GAV. 1992. Psychophysical basis of perceived exertion. Med Sci Sports Exerc, 14:377-81.

Carone M, Bertolotti G, Anchisi, et al. 1999. II St. George's Respiratory Questionnaire (SGRQ): la versione italiana. Rass Pat App Respir, 14:31-7.

Casaburi R, Kukafka D, Cooper CB, et al. 2005. Improvement in exercise tolerance with the combination of tiotropium and pulmonary rehabilitation in patients with COPD. Chest, 127:809-17. 
Casaburi R, Patessio A, Ioli F, et al. 1991. Reduction in exercise lactic acidosis and ventilation as a result of exercise training in patients with obstructive lung disease. Am Rev Respir Dis, 143:9-18.

Celli BR, MacNee W, Agusti A, et al. 2004. Standards for the diagnosis and treatment of patients with COPD: a summary of the ATS/ERS position paper. Eur Respir J, 23:932-46.

Domingo-Salvany A, Lamarca R, Ferrer M, et al. 2002. Health-related quality of life and mortality in male patients with chronic obstructive pulmonary disease. Am J Respir Crit Care Med, 166:680-5.

Foglio K, Bianchi L, Bruletti G, et al. 1999. Long-term effectiveness of pulmonary rehabilitation in patients with chronic airway obstruction. Eur Respir J, 13:125-32.

Foglio K, Bianchi L, Bruletti G, et al. 2007. Seven-year time course of lung function, symptoms, health-related quality of life, and exercise tolerance in COPD patients undergoing pulmonary rehabilitation. Respir Med, 101:1961-70.

Georgiadou O, Vogiatzis I, Stratakos G, et al. 2007. Effects of rehabilitation on chest wall volume regulation during exercise in COPD patients. Eur Respir J, 29:284-91.

Kesten S, Casaburi R, Kukafka D, et al. 2008. Improvement in self-reported exercise participation with the combination of tiotropium and rehabilitative exercise training in COPD patients. J Chron Obstruct Pulmon Dis, 3:127-36.

Ketelaars CAJ, Schlosser MAG, Mostert R, et al. 1996. Determinants of health-related quality of life in patients with chronic obstructive pulmonary disease. Thorax, 51:39-43.

Mahler DA, Weinberg DH, Wells CK, et al. 1984. Baseline dyspnoea index and Transition dyspnoea index: the measurement of dyspnoea. Chest, 85:751-8.

Maltais F, Hamilton A, Marciniuk D, et al. 2005. Improvements in symptom-limited exercise performance over $8 \mathrm{~h}$ with once-daily tiotropium in patients with COPD. Chest, 128:1168-78.

Maltais F, LeBlanc P, Simard C, et al. 1996. Skeletal muscle adaptation to endurance training in patients with chronic obstructive pulmonary disease. Am J Respir Crit Care Med, 154:442-7.
Mannino DM, Buist S. 2007. Global burden of COPD: risk factors, prevalence, and future trends. Lancet, 370:765-73.

Nici L, Donner C, Wouters E, et al; on behalf of the ATS/ERS pulmonary rehabilitation writing committee. 2006. American Thoracic Society/ European Respiratory Society statement on pulmonary rehabilitation. Am J Respir Crit Care Med, 173:1390-413.

Nishimura K, Izumi T, Tsukino M, Oga T. 2002. Dyspnea is a better predictor of 5-year survival than airway obstruction in patients with COPD. Chest, 121:1434-40.

O'Donnell DE, Fluge T, Gerken F, et al. 2004. Effects of tiotropium on lung hyperinflation, dyspnea and exercise tolerance in COPD. Eur Respir J, 23:832-40.

Pepin V, Brodeur J, Lacasse Y, et al. 2007. Six-minute walking versus shuttle walking: responsiveness to bronchodilation in chronic obstructive pulmonary disease. Thorax, 62:291-8.

Pitta F, Troosters T, Spruit MA, et al. 2005. Characteristics of physical activities in daily life in chronic obstructive pulmonary disease. Am J Respir Crit Care Med, 171:972-7.

Quanjer PH. 1983. Standardization of lung function test of the European Community for Coal and Steel. Bull Eur Physiopathol Respir, 19(Supp15):7-10.

Redelmeier DA, Bayoumi AM, Goldstein RS, et al. 1997. Interpreting small differences in functional status: the six minute walk test in chronic lung disease patients. Am J Respir Crit Care Med, $155: 1278-82$.

Saey D, Maltais F. 2005. Role of peripheral muscle function in rehabilitation. In: Donner CF, Ambrosino N, Goldstein RS (eds). Pulmonary rehabilitation. London: Arnold Pub, pp. 80-90.

Tsukino M, Nishimura K, Ikeda A, et al. 1996. Physiologic factors that determine the health related quality of life in patients with COPD. Chest, 110:896-903.

Turner SE, Eastwood PR, Cecins NM, et al. 2004. Physiologic responses to incremental and self-paced exercise in COPD: a comparison of three tests. Chest, 126:766-73. 